\section{Online archive must serve authors as well as publishers}

Sir - In an extremely important, timely and welcome development for science, the E-Biomed proposal has evolved into PubMed Central, a free online public archive of the peer-reviewed and nonpeer-reviewed literature in biology. It will be launched by the US National Institutes of Health (NIH) next January (Nature 401, $6 ; 1999)$. There is only one fundamental question that needs to be answered about the revised proposal: will authors be able to self-archive their refereed articles in PubMed Central?

The revised proposal is not clear about this question: it could be that only publishers will be able to archive refereed papers. This would be regrettable, because publishers are not likely to want to give away papers free, whereas authors are.

If authors are allowed to self-archive their refereed articles, PubMed Central will not only quickly make the biological literature into the optimal free resource for biological science, but it will provide a model for adoption by all other learned disciplines.

The director of the NIH, Harold Varmus, says that PubMed Central will be a web-based repository for barrier-free access to primary reports in the life sciences. Assuming that "barrier-free" means free for one and all in perpetuity, this will be an invaluable contribution to the advancement of biological and medical research.

Varmus also says that the screening of non-peer-reviewed reports will be the responsibility of groups that have no direct relationship to the NIH. This is as it should be. Peer review should continue to be implemented by scientific publishers and societies, and reports should be provided to PubMed Central from participating publishers and societies that have mediated the review process. But what about peerreviewed reports from non-participating publishers and societies? Will the authors of such work be able to archive it in PubMed Central too? Or will the work available for free for all in PubMed only be that published by 'participating' publishers and societies?

The non-peer-reviewed reports will also enter PubMed Central through independent organizations, which will be responsible for screening this material. Will authors be able to self-archive their non-peer-reviewed reports? Some screening is a prudent idea but it must not be so restrictive as to prevent the self-archiving of preprints that are being submitted to peer-reviewed journals.

Will the availability of the peer-reviewed literature online free for all be conditional only on the active collaboration of publish- ers (who currently derive their revenue from selling it) or also on the active collaboration of authors (who give it away)?

Varmus has asked publishers, societies, editorial boards and other organizations interested in depositing content in PubMed Central to contact him at PubMedCentral@nih.gov.Butwhat about authors interested in depositing their peer-reviewed and non-peer-reviewed reports? Is, say, university affiliation sufficient (which would be a good first step), or is it still to be only publishers who determine whether or not their authors' freely given reports can be given away for free? A great deal rests on the answer to this question.

It is to be hoped that, as PubMedCentral accrues more and more of the literature and makes it available to everyone for free, the bioscience community will become as addicted to this online archive as the physics community has become to the Los Alamos archives. In that case, the freeing of the rest of the literature will not lag far behind.

An online debate on this topic is at http://amsci-forum.amsci.org/archives/ september98-forum.html

Stevan Harnad

Department of Electronics and Computer Science, University of Southampton,

Highfield, Southampton SO17 1BJ, UK

\section{A view from Kansas on that evolution debate}

Sir - I have recently attended two lectures in the wake of the controversial decision by the Kansas State Board of Education to "eliminate" the required teaching of evolution (see Nature 400, 701; 1999). Philip Johnson, a professor of law at the University of California, Berkeley, and John Staver, cochair of the committee responsible for drafting the new Kansas standards - and whose draft had been, according to him, "severely edited" by the board to "remove evolution" — both presented their definitions of science and evolution to sympathetic audiences. Both erroneously presented what they believed to be the other party's definitions of these concepts.

The crucial difference between what the creationists believe and what the proponents of evolutionary theory accept concerns the issue of whether the origins of life were driven by randomness or by an intelligent creator. Many creationists are supportive of scientific enquiry for biblical reasons such as in Romans 1:20, "For since the creation of the world God's invisible qualities, his eternal power and divine nature, have been clearly seen, being understood from what has been made".

Creationists, according to Johnson, do not doubt that DNA encodes the features of an organism or that changes in DNA (mutations) give rise to variation in those features which are subject to selective pressures in nature. Mainstream creationists also accept that genetic and phenotypic changes could result in speciation. They consider evolution as a plausible model to account for the natural history of living things, but they see a great distinction between the empirically proven elements of evolution (micro-evolution) and the explanation of speciation and origins of life (macro-evolution). Students in Kansas will still be required to learn the former, but it will be left to local school districts to decide whether they are required to learn the latter.

The lesson to be learned from the events in Kansas is that science educators everywhere must do a better job of teaching evolution. It must be made clear that the evidence supporting the mechanism of evolution is empirical and proven, but that speciation and natural history are derived from the admittedly weaker evidence of observation. The fact that one cannot reproduce the experiment does not diminish the validity of macro-evolution, but the observed phenomena supporting the theory must be presented more clearly.

Additionally, one must question the interpretations of the observed phenomena and discuss the weaknesses of the model. Honest scientists are far more inspiring than defensive ones who scoff arrogantly at the masses and fear that discussing the problems of macro-evolutionary theory will weaken general acceptance of it. On the contrary, free debate is more likely to encourage the curious to seek solutions. Most important, it should be made clear in the classroom that science, including evolution, has not disproved God's existence because it cannot be allowed to consider it (presumably).

Even if all the data point to an intelligent designer, such an hypothesis is excluded from science because it is not naturalistic. Of course the scientist, as an individual, is free to embrace a reality that transcends naturalism.

Scott C. Todd

Department of Biology, Kansas State University, 18 Ackert Hall, Manhattan, Kansas 66506, USA

\section{Wellcome for education on science in society}

Sir - You have reported criticisms of the new science centre, Explore at Bristol (Nature 400, 801 \& 804; 1999; see also response from Gillian Thomas, Nature 401, 111-112; 1999). There is currently a great deal of public criticism about aspects of medical research which needs to be addressed by those involved. The 
Wellcome Trust spends about $£ 400$ million (US\$640 million) a year on research, and has committed roughly $£ 34$ million of this to science centres and museums in the United Kingdom, including Bristol. Why?

It is crucial to explain to the public the excitement of medical research and its potential benefits without ignoring its social impact and implications. The Wellcome Trust supports initiatives such as Explore at Bristol precisely because they aim to go beyond the traditional 'hands-on' approach to explore the wider context within which biomedical science develops.

Traditional 'hands-on' science centres barely touch modern science or biomedicine, and the social context is ignored altogether. Although hands-on exhibits are powerful learning tools, they are not necessarily the best way to tackle modern biology or the social issues raised by the human genome project, for example. Yet the general public has to be informed about the place of science in society if it is to trust science and scientists.

This task is by far the most important facing the scientific community at present and the new science centres will be crucial in this regard. There may indeed be teething troubles, not least owing to the very short timescales over which these huge projects have had to develop. But the Wellcome Trust's interest is long term, not only helping to build on the solid foundations of past innovation, but also aiming to experiment with new directions.

Laurence Smaje

The Wellcome Trust, 183 Euston Road, London NW1 2BE, UK

\section{Confidentiality is vital to bioweapons control}

Sir-Biological weapons still form part of the world's arsenal, as the work of the United Nations Special Commission (UNSCOM) in Iraq has underlined ${ }^{1}$. A weapons control system based on UN Security Council resolutions implies serious threats not just to national sovereignty but also to confidential information - an important concern in today's competitive academic and industrial environment. Since a treatybased inspection regime relies on the willingness of states to sign the treaty, they have to be certain that their confidential information will remain secure.

Current negotiations in Geneva for a protocol to strengthen the biological weapons convention ${ }^{2}$ are likely to lead to a combined reporting-inspection system. As in the chemical weapons convention ${ }^{3}$, site inspections and inclusion of non-military sites are being discussed: two elements with far-reaching consequences for confidentiality issues. In addition to classical confidentiality provisions (such as guidelines, a commission, individual secrecy agreements, and restricted access to information), the selection of appropriate triggers for countries to make declarations under the protocol should weed out threats to the loss of confidential information.

The draft protocol confines required declarations of research and development to listed agents and toxins, biological defence and maximum containment facilities. The current talks are still discussing the scope of inspections and when they should be held ${ }^{2}$ - for example, should they be random? Inspections will be based on mandates defining the purpose of the inspection, ranging from confidencebuilding, auditing and clarifying information to investigating a suspected breach of the biological weapons convention.

Until now, concerns about the risk to confidential information have not been substantiated by declarations based on the current draft protocol and several practice inspections.

The trend to incorporate private institutions can also be seen in other international agreements, such as the Convention on Access to Information, Public Participation in Decision-making and Access to Justice in Environmental Matters ${ }^{4}$ and the draft Biosafety Protocol to the Convention on Biological Diversity ${ }^{5}$.

In order to increase public and political acceptance of biological research and biotechnology, confidentiality issues and the need for more transparency will have to be brought into balance.

Johannes Rath*, Bernhard Jank $\dagger$, Otto Doblhoff-Dier $\dagger$

${ }^{*}$ Institute for Zoology, University of Vienna,

Althanstrasse 14, A-1090 Vienna, Austria

$\dagger$ Institute of Applied Microbiology,

University of Agriculture,

Nussdorfer Lände 11, A-1190 Vienna, Austria

1. Seelos, C. Nature 398, 187-188 (1999).

2. http://www.brad.ac.uk/acad/sbtwc/

3. http://www.opcw.nl/cwc/cwc-eng.htm

4. http://www.unece.org/env/europe/ppconven.htm

5. http://www.biodiv.org/biosafe/bswg6/bswg6.html\#excop

\section{Devil in the detail}

Sir - William Thompson [sic] and Lord Kelvin are credited with naming Maxwell's demon in Seth Lloyd's obituary of Rolf Landauer (Nature 400, 720; 1999). The demon, or at least an editorial gremlin, is having a little joke: William Thomson (without the 'p') and Lord Kelvin were one and the same, transmuted by act of Queen Victoria in 1892.

\section{Nicholas J. Cox}

Department of Geography, University of Durham, Durham DH1 3LE, UK

\section{Turning the tide}

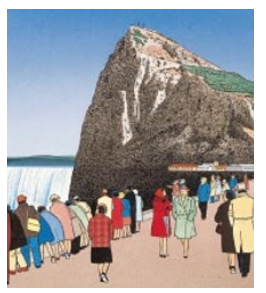

Sir - A cartoon in News and Views seems to prove that 5.33 million years ago the Mediterranean emptied into the Atlantic rather than filled from the

View from the Rock: Atlantic (Nature 400, how it should look. 613; 1999). Must we reverse our theories,

or should you reverse your slide?

Tim Robinson

Folding Landscapes, Roundstone, Connemara, Co. Galway, Ireland

\section{Medicine and biology are more than biomedicine}

Sir - While I concur with Ken Dill's call for increased support of research in physics, chemistry, mathematics and computer science, I am troubled by his reasoning ${ }^{1}$. He conflates biology and medicine into an ill-defined hybrid "biomedicine", which he believes is reducing "the problems of disease to problems of molecular science". I believe this belittles both biology and medicine.

The biological sciences are quite distinct from medicine. Obviously they overlap, but so do each of them with the other disciplines that Dill mentions. Further, advances in biology and medicine feed back into and stimulate what he terms the "basic sciences", and may lead to whole new research paradigms ${ }^{2}$.

The unidirectional model of "basic" and "applied" research implied by the pyramid in his Fig. 1 derives less from his view of their interactions than from the rapid growth of the budget of the US National Institutes of Health (which supports "biomedicine") compared with US agencies concerned with the physical sciences.

Many research programmes could influence human health and need new, long-term funding. Among these, I would stress patient-oriented medical research itself $^{3}$, including human pathophysiology, epidemiology, pharmacology, clinical trials and health-services research. A good case could also be made for behavioural, population and other as yet 'soft' sciences. If Dill wishes to present a two-dimensional model for the relationships of the sciences to the curing of disease, I would suggest a circle with radiating spokes for the many disciplines that need increased support.

Alan N. Schechter

5405 Beech Avenue, Bethesda, Maryland 20814, USA

1. Dill, K. A. Nature 400, 309-310 (1999).

2. Gershon, E. Persp. Biol. Med. 42, 95-102 (1998).

3. Goldstein, J. L. \& Brown, M. S. J. Clin. Invest. 99, 2803-2811 (1997). 